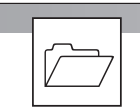

\title{
Primary Burkitt lymphoma of the thyroid gland in a 28-year-old female
}

\author{
Francesk Mulita ${ }^{1}$, Elias Liolis ${ }^{2}$, Ioannis Maroulis' ${ }^{1}$ Konstantinos Panagopoulos ${ }^{1}$, Kerasia-Maria Plachouri ${ }^{3}$ \\ ${ }^{1}$ Department of Surgery, General University Hospital of Patras, Patras, Greece \\ ${ }^{2}$ Division of Oncology, Department of Medicine, General University Hospital of Patras, Patras, Greece \\ ${ }^{3}$ Dermatology Department, General University Hospital of Patras, Patras, Greece
}

Key words: thyroid abscess; ethanol ablation; thyroidectomy; thyroid nodule

Burkitt lymphoma (BL) of the thyroid gland is an aggressive and very rare $\mathrm{B}$ cell non-Hodgkin lymphoma (NHL), and comprises approximately $2 \%$ of thyroid lymphomas (TLs) [1]. TL can be classified as either primary or secondary TL. There have been a few isolated cases of primary Burkitt lymphoma (PBL) of the thyroid gland in the adult population [2].

A 28-year-old female without a significant past medical history was admitted to our clinic with a visible swelling on the right side of the neck. On examination she was found to have a small right-sided firm thyroid swelling, with no lymphadenopathy. At that time blood tests as well as thyroid-stimulating hormone levels were within the normal range. Ultrasonography (US) was performed and revealed a mass $1.5 \times 1 \mathrm{~cm}$ in the lower pole of right lobe of the thyroid. Fine needle aspiration cytology (FNAC) of the mass was done resulting in suspicion of NHL. A thoraco-abdominal computed tomography (CT) scan showed a hypodense lesion in the right lobe of the thyroid gland measuring $2 \times 1.5 \times 1 \mathrm{~cm}$ in size (Fig. 1). PET-CT examination was not considered. The patient underwent right thyroid lobectomy with isthmectomy (Fig. 2, 3). An intraoperative histopathological examination was not conducted. She did not have any postoperative complications and was discharged on the fourth postoperative day in good clinical condition. A surgical specimen of thyroid was received for histological examination and was suggestive of chronic thyroiditis.

The patient presented with a rapidly growing neck mass and dysphagia 35 days after surgery. On examination neck pain and facial oedema was recognized. A cervical spine X-ray as well as cervical magnetic resonance imaging (MRI) showed tracheal

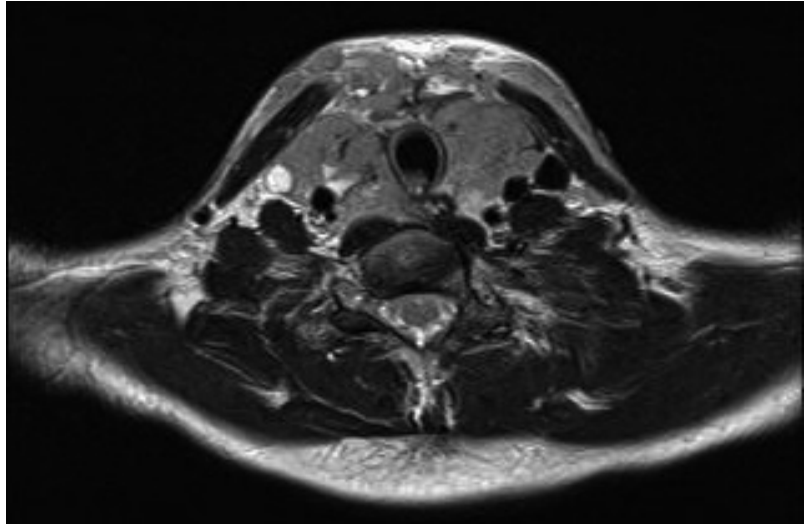

Figure 1. CT scan showing a hypodense lesion in the right lobe of the thyroid gland

compression (Fig. 4 A, B) by enlarged cervical lymph nodes. A biopsy of the cervical mass was taken, and the histological examination showed primary thyroid Burkitt lymphoma (PTBL). On microscopic examination, the tumour cells were composed of diffuse sheets of lymphoid cells admixed with scattered tangible body type macrophages. Immunohistochemical staining was performed and the tumour cells were positive for L26, CD79a, CD10, bcl-6, Ki-67 (100\%), myc (95\%), FOXP1, and CD5 and negative for bcl-2,TdT, CD34, D1, SOX-11, MUM-1, CD138, CD43, and CD3. Immunohistochemistry for Ebstein-Barr virus (EBV) was negative. The final diagnosis of PTBL was confirmed on fluorescence in situ hybridization (FISH), which showed tumour cell positivity for the $t(8 ; 14)$ translocation. The patient was transferred to the haematology clinic to receive chemotherapy (rituximab, cyclophosphamide, doxorubicin, vincristine and prednisolone) as the tumour was catagorised as stage 1 . 


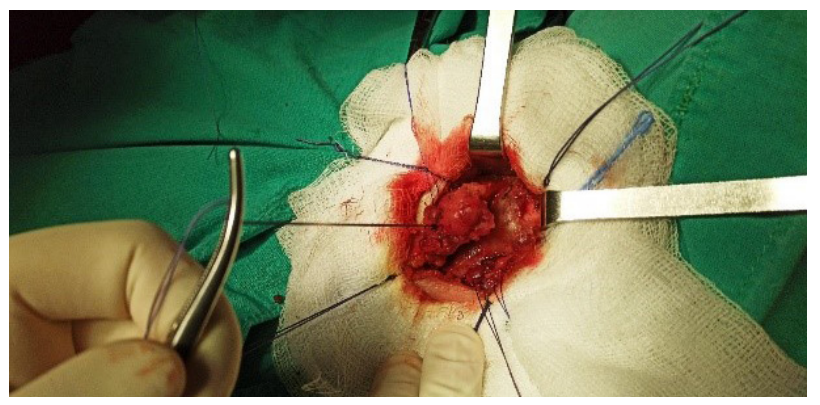

Figure 2. Application of a cervical low incision in the thyroidectomy with the protection of the skin

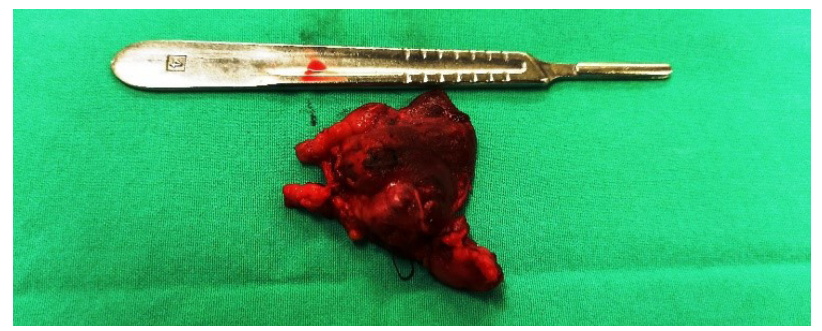

Figure 3. Surgical specimen of the right thyroid lobectomy with isthmectomy

Primary thyroid lymphoma is a very uncommon pathological entity that accounts for less than $5 \%$ of all thyroid carcinomas, and Burkitt lymphoma comprises less than $2 \%$ of thyroid lymphomas. Primary Burkitt thyroid lymphoma (PBTL) typically occurs in middle-aged to older females in the setting of autoimmune thyroiditis. Hashimoto's thyroiditis and lymphoma of the thyroid co-exist in $40-80 \%$ of the patients [1]. PBTL is endemic in Africa (associated with viral infections, particularly EBV) and sporadic in other parts of the world [3]. Burkitt lymphoma is one of the first malignant tumours shown to have a chromosomal translocation that activates the MYC oncogene, which normally resides on chromosome 8q24 [4]. Sometimes lymphomas originating in the thyroid can mimic anaplastic carcinoma, because they both have rapid growth, which can cause obstructive symptoms such as dyspnoea, dysphagia, hoarseness of voice, and pain. Medical treatment is considered the principal therapeutic method for primary thyroid lymphoma, and surgery is generally diagnostic.

We describe a rare case of a primary thyroid Burkitt lymphoma in a 28-year-old female. In this case FNAC was suspicious of NHL. The patient underwent right thyroid lobectomy with isthmectomy, because the likelihood of other types of thyroid cancer could not be excluded. However, the pathological exam after surgery was negative for malignancy. The diagnosis was confirmed by a second biopsy. A recent review of the English literature revealed very few cases of thyroid Burkitt lymphoma. However, this is the first case that

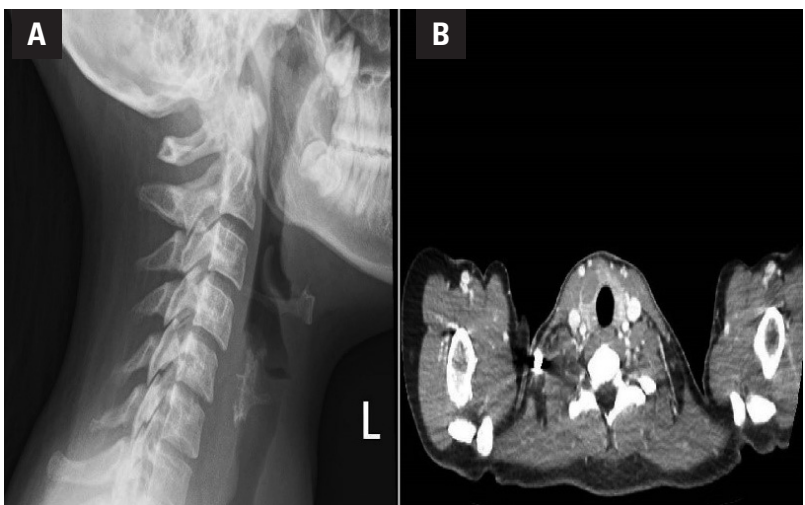

Figure 4. The cervical spine X-ray $(A)$ and cervical magnetic resonance imaging $(\boldsymbol{B})$ showing tracheal compression by enlarged cervical lymph nodes

diagnosis was not made after surgery. BL of the thyroid gland is an aggressive and very rare NHL, and accurate diagnosis is critical for the assessment of the stage of the disease and the selection of the appropriate therapy. Regarding the therapy, doctors have to decide based on the histology of the disease, the anatomical stage, and the presence of poor prognostic features. Patients with early-stage lymphoma at the thyroid gland are usually treated with chemotherapy with or without radiotherapy. Surgical therapy is required only in the cases of symptoms that cause pain and affect the airways. It should also be noted that total thyroidectomy is proposed for patients at later stages of the disease.

\section{Informed consent}

Informed consent was obtained from the patient for the publication of this article.

\section{Conflict of interest}

None declared.

\section{Funding sources}

None declared.

\section{Authors' contributions}

All authors contributed equally to the article.

\section{References}

1. Hayashi T, Hirokawa M, Kuma S, et al. Clinicopathological features of primary thyroid Burkitt's lymphoma: a systematic review and meta-analysis. Diagn Pathol. 2020; 15(1): 13, doi: 10.1186/s13000-020-00933-z, indexed in Pubmed: 32035483

2. Cooper K, Gangadharan A, Arora RS, et al. Burkitt lymphoma of thyroid gland in an adolescent. Case Rep Pediatr. 2014; 2014: 187467, doi: 10.1155/2014/187467, indexed in Pubmed: 24868478.

3. Allaoui M, Benchafai I, Mahtat ElM, et al. Primary Burkitt lymphoma of the thyroid gland: case report of an exceptional type of thyroid neoplasm and review of the literature. BMC Clin Pathol. 2016; 16: 6, doi: 10.1186/s12907-016-0028-6, indexed in Pubmed: 27175103.

4. Kalisz K, Alessandrino F, Beck R, et al. An update on Burkitt lymphoma: a review of pathogenesis and multimodality imaging assessment of disease presentation, treatment response, and recurrence. Insights Imaging. 2019; 10(1): 56, doi: 10.1186/s13244-019-0733-7, indexed in Pubmed: 31115699. 